\title{
Association between dietary fatty acids pattern and incidence of oral cancer: A case-control study from Southeast China
}

\section{Yi Fan}

Fujian Medical University

\section{Yu Qiu}

First Affiliated Hospital of Fujian Medical University

\section{Qing Chen}

Fujian Medical University

\section{Sijie Wang}

Fujian Medical University

\section{Mingming $\mathrm{Xu}$}

Fujian Medical University

\section{Yanni Li}

Fujian Medical University

\section{Yanfeng Weng}

Fujian Medical University

Jiawen Qian

Fujian Medical University Jing Wang

Fujian Medical University

Fa Chen

Fujian Medical University

Jing Wang

Fujian Medical University

\section{Bin Shi}

First Affiliated Hospital of Fujian Medical University

\section{Lizhen Pan}

First Affiliated Hospital of Fujian Medical University

\section{Lisong Lin}

First Affiliated Hospital of Fujian Medical University

\section{Baochang He}

Fujian Medical University

Fengqiong Liu ( $\square$ lfq@fjmu.edu.cn ) 
Fujian Medical University https://orcid.org/0000-0001-7786-7305

\section{Research}

Keywords: fatty acid pattern, saturated fatty acids, oral cancer, principal component analysis, casecontrol study

Posted Date: July 27th, 2021

DOl: https://doi.org/10.21203/rs.3.rs-713275/v1

License: (a) (i) This work is licensed under a Creative Commons Attribution 4.0 International License. Read Full License 


\section{Abstract}

Objective

To investigate the relationship between dietary fatty acid pattern and the risk of oral cancer (OC).

Method

In 446 patients with primary oral cancer and 448 controls, we assessed prediagnosis consumption of 159 food items by food frequency questionnaires completed within 1 week of diagnosis. Fatty acid patterns were identified using principal component analysis. Odds ratio $(O R)$ and $95 \%$ confidence $(C l)$ interval was calculated.

Results

General differences of fatty acid intake were observed between case and control, intake of saturated fatty acids such as C14:0, C16:0, C18:0 are higher in case than control group $(p<0.001)$, intake of monounsaturated fatty acid such as C18:1 is higher in case than control group $(p<0.001)$. We identified a fatty acid pattern which was characterized by saturated fatty acids and it explained $33.2 \%$ of the overall variability of the 32 fatty acids. The identified fatty acid pattern scores were positively associated with a higher risk of oral cancer [multivariable-adjusted OR comparing extreme quintiles, 3.325 (95\% Cl: 2.222, 4.975); $\left.P_{\text {trend }}<0.001\right]$.

Conclusions

General differences of dietary fatty acids were observed between oral cancer patients and controls. A dietary fatty acids pattern, which was mainly composed of saturated fatty acids were positively correlated with the risk of oral cancer.

\section{Introduction}

Oral cancers $(\mathrm{OC})$ is one of the most common cancers in head and neck cancers with nearly 40,000 new cases recognized in China in 2015[1]. According to current statistics from the GLOBOCAN 2018, the incidence and mortality of oral cancers in China in 2018 was 2.0/100,000 and 0.97/100,000 respectively [2]. Commonly recognized etiologic factors of oral carcinoma include smoking, drinking, oral hygiene, HPV, betel quid consumption[3-9]. In addition to the above mentioned traditional risk factors, other environmental exposures such as dietary factors were also reported in recent studies[10-12]. Among dietary factors, role of fatty acids in tumorigenesis has got increased interests.

Fatty acids, including saturated fatty acid (SFA), n-3 and n-6 poly unsaturated fatty acid (PUFA), trans fatty acid(TFA), have been reported associated with the risk of varied types of cancer such as prostate cancer[13,14], pancreatic cancer[15, 16], colorectal cancer[17, 18], lung cancer[19]. However, reports on the association between fatty acid and head and neck tumors, especially oral cancer, are rare. 
Most studies have considered individual fatty acids as separate exposures. However, individual fatty acids tend to be correlated with each other, partially due to shared food sources and metabolic pathways. Humans consume foods consisting of a range of meals with complex mixtures of fatty acids that are probably to be interactive or synergistic[20,21]. Because of the complexity of diet and highly interrelated nature of dietary exposures, analyses of individual fatty acid and foods often ignore the many potential interactions between components of a diet and disease risk [22]. Hence, in addition to examining individual fatty acids, analyzing fatty acids as sets of patterns could capture these complexities and account for these technical issues, while shedding light on the biological interactions between different fatty acids and their relation with disease risk[23, 24].

Therefore, we performed a case-control study to identify potential dietary fatty acid patterns and elucidate their role in the development in oral cancer.

\section{Materials And Methods}

\section{Study design and population}

From September 2016 and December 2020, a case-control study was conducted in Fujian province, China. As previously described[25], oral cancer patients were recruited from the First Affiliated Hospital of Fujian Medical University. The inclusion criteria of the patients were as follows: (1) Histologically confirmed primary oral cancer; (2) Chinese Han population and residence in Fujian Province; (3) Age above 18 years old. Patients with second primary, recurrent or metastasized cancer, and previous radiotherapy or chemotherapy were excluded. Control participants were recruited from the health examination center of the same hospital during the same period. Those with history of cancer were excluded. Additionally, we excluded: those who have extreme daily caloric intake $(>4200 \mathrm{Kcal}$ or $<700$ Kcal for man; $>3500 \mathrm{Kcal}$ or $<500 \mathrm{Kcal}$ for woman). Finally 446 patients and 448 control participants were admitted to the study.

All participants provided a signed informed consent, and the study protocol was approved by the Institutional Review Board of Fujian Medical University (Approval number: 2011053; Approval date: March 10, 2011) and conducted in accordance with the ethical standards described in the Declaration of Helsinki.

\section{Data Collection}

A structured questionnaire was used to collect information through face to face interviews conducted by well-trained interviewers. The questionnaire included socio-demographic characteristics, dietary intake, tobacco smoking and alcohol drinking status, oral hygiene, family history of cancer etc.

Body mass index (BMI) was calculated as weight (in kilograms) divided by the square of the height (in meters). Subjects who had smoked at least 100 cigarettes during their lifetime were considered tobacco 
smokers. Educational level was coded as low (lower vocational training or primary school), or high (secondary school and above).Alcohol drinker was defined as consumed at least one drink per week and lasting for more than 6 months continuously[26]. A complete description of the oral hygiene score is available in our previous study[7]. Oral hygiene score $=$ teeth brushing + the number of missing teeth + wearing dentures + regular dental visits + recurrent dental ulceration. The range of oral hygiene score was $0-5$, and higher score indicated worse oral hygiene.

Information on habitual dietary intake was gathered using a validated semiquantitative Food Frequency Questionnaire (FFQ) at baseline. The dietary data during 1 year prior to the diagnosis for cases or the year prior to interview for controls were selected. The questionnaire contained 8 broad categories (grains; beans and soy products; vegetables; fruits; animal food; algal fungi and nuts; beverages and soup; fried foods and pickled foods) and 17 sub-categories (grains; beans and soy products; dark vegetables; light color vegetables; purple vegetables; fresh beans; fruits; livestock; poultry; fish; processed meat; red meat; eggs; dairy; algal fungi and nuts; fried foods; pickled foods), and the total number of food items is 159. For each food item, participants were asked how often, on average, over the previous year, they had consumed the food, according to a commonly used unit or portion size. The intake of nutrient and dietary fatty acids in food of each item $=$ the intake of food of each item $(\mathrm{g} / \mathrm{d}) \times$ the content of dietary fatty acids and nutrients in the edible part of the food $(100 \mathrm{~g}) / 100 \mathrm{~g}$. The edible food part, and the content of fatty acids and nutrients reference to 2009 "China Food Composition Tables".

\section{Statistical analysis}

Intakes of energy and nutrients were log-transformed and then fatty acids intakes were adjusted for energy intake using the residuals method[27]. Quantitative data were presented as median with interquartile range, while qualitative variables were presented as frequency (numbers and percentages). Fatty acids patterns were derived using principal component analysis (PCA) based on 32 fatty acids. Principal components were inferred as representing fatty acid patterns. The pattern matrix from PCA was then used to calculate the scores and categorized into tertiles, and the lowest tertiles was used as the reference. The first principal component was submitted for further analyses to provide potential biological implications of this single combination of fatty acids. This removed the need for subjective decisions about how many components to derive, which matrix rotation method to use, and how to account for multiple testing.

Chi-square tests were used to compare the main characteristics between cases and controls. The distribution of dietary fatty acids was analyzed using Wilcoxon rank sum test. Pearson correlation coefficients were calculated, and a hierarchical cluster tree and heatmap were generated to visually assess correlation between fatty acids[28]. Associations between fatty acids pattern score and intakes of 17 food groups, and macronutrients were assessed by spearman correlation analysis. Fatty acids pattern score was evaluated categorically and continuously in the logistic regression model, and ORs and their 95\% Cls were calculated. In addition, fatty acids pattern score was evaluated continuously by restricted cubic splines. 
All analyses were performed using R software (version 4.0.3), with 2-tailed $p$ values $<0.05$ considered as statistically significant.

\section{Result}

\section{Characteristics of the study population}

The baseline characteristics and main lifestyle factors for cases and controls are shown in Table 1. Compared with controls, cases were more likely to have a higher proportion of tobacco, alcohol consumption, tumor history and worse oral hygiene. In addition, the distribution of gender, education level, BMI, residence was significantly different between the case and control group $(p<0.05)$. General differences of fatty acid intake were observed between case and control, intake of SFA such as C14:0, C16:0, C18:0 are higher in case than control group ( $p<0.001)$, intake of monounsaturated fatty acids(MUFA) such as $\mathrm{C} 18: 1$ is higher in case than control group $(p<0.001)$. The distribution of dietary fatty acids between the case and control are shown in Supplement Figure 1.

Table1 Characteristics of the case $(n=446)$ and control $(n=448)$ 


\begin{tabular}{|c|c|c|c|}
\hline Variable & Case & Control & $P$ \\
\hline Age & & & $<0.001$ \\
\hline$\varangle 49$ & $94(21.1 \%)$ & $210(46.9 \%)$ & \\
\hline$\geq 49$ & $352(78.9 \%)$ & $238(53.1 \%)$ & \\
\hline Gender & & & 0.002 \\
\hline Male & $258(57.8 \%)$ & $213(47.5 \%)$ & \\
\hline Female & $188(42.2 \%)$ & $235(52.2 \%)$ & \\
\hline Education & & & $<0.001$ \\
\hline low & $77(17.3 \%)$ & $204(45.5 \%)$ & \\
\hline high & $369(82.7 \%)$ & $244(54.5 \%)$ & \\
\hline Marital status & & & 0.699 \\
\hline Married & $408(91.5 \%)$ & $413(92.2 \%)$ & \\
\hline Unmarried and Others & $38(8.5 \%)$ & $35(7.8 \%)$ & \\
\hline BMI & & & 0.024 \\
\hline$<18.5$ & $39(8.7 \%)$ & $19(4.2 \%)$ & \\
\hline 18.5区 & $284(63.7 \%)$ & $297(66.3 \%)$ & \\
\hline$\geq 24$ & $123(27.6 \%)$ & $132(29.5 \%)$ & \\
\hline Residence & & & 0.008 \\
\hline Country & $258(57.8 \%)$ & $298(66.5 \%)$ & \\
\hline City & $188(42.2 \%)$ & $150(33.5 \%)$ & \\
\hline Occupation & & & 0.231 \\
\hline Farmers and Workers & $148(33.2 \%)$ & $132(295 \%)$ & \\
\hline Others & $298(66.8 \%)$ & $316(66.8 \%)$ & \\
\hline Tobacco smoking & & & 0.001 \\
\hline No & $259(58.1 \%)$ & $307(68.5 \%)$ & \\
\hline Yes & $187(41.9 \%)$ & $141(31.5 \%)$ & \\
\hline Alcohol drinking & & & $<0.001$ \\
\hline No & $294(65.9 \%)$ & $349(77.9 \%)$ & \\
\hline Yes & 152(34.1\%) & $99(22.1 \%)$ & \\
\hline
\end{tabular}




\begin{tabular}{|llll|}
\hline Tumor history & & & $<0.001$ \\
\hline No & $374(83.9 \%)$ & $413(92.2 \%)$ & \\
\hline Oral hygiene score & $72(16.1 \%)$ & $35(7.8 \%)$ & \\
\hline $0-2$ & & & $<0.001$ \\
\hline $3-5$ & $79(17.7 \%)$ & $167(37.3 \%)$ & \\
\hline $6-8$ & $257(57.6 \%)$ & $248(55.4 \%)$ & \\
\hline
\end{tabular}

\section{Identification of fatty acid patterns}

By applying PCA, we found 4 principal components which could explain $75.7 \%$ of the variance of the dietary fatty acid consumption, as the scree plot was shown in Figure 1. And we chose the first principal component because it explained $33.2 \%$ of the variation of 32 fatty acids. The components is characterized by saturated fatty acid (the "SFA" pattern), which mainly included octanoic acid (C8:0), undecanoic acid (C11:0), lauric acid (C12:0), myristic acid (C14:0), pentacarbonate (C15:0), and (C16:1) in MUFA. Factor loadings of the individual fatty acids in the "SFA" patterns are shown in Table 2.

Additionally, correlation analysis among individual fatty acids were performed, and heatmap were derived using correlation coefficients among individual fatty acids. A similar pattern was identified in cluster analysis, as fatty acids adjacent in the tree had similar loading values (Figure 1).

Table 2 Factor loading of the individual fatty acids in the FA pattern 


\begin{tabular}{|c|c|c|}
\hline Type of fatty acid & Name & Loading of the FA pattern * \\
\hline \multicolumn{3}{|c|}{ Saturated fatty acids } \\
\hline 6:0 & Caproic & 0.334 \\
\hline 8:0 & Caprylic & 0.731 \\
\hline 10:0 & Capric & 0.596 \\
\hline 11:0 & Undecanoic & 0.702 \\
\hline 12:0 & Lauric & 0.783 \\
\hline 13:0 & Tridecanoic & 0.618 \\
\hline 14:0 & Myristic & 0.848 \\
\hline 15:0 & Pentadecanoic & 0.720 \\
\hline 16:0 & Palmitic & 0.787 \\
\hline 17:0 & Heptadecanoic & 0.525 \\
\hline 18:0 & Stearic & 0.792 \\
\hline 19:0 & Nonadecanoic & 0.586 \\
\hline 20:0 & Arachidic & 0.559 \\
\hline 22:0 & Behenic & 0.037 \\
\hline \multicolumn{3}{|c|}{ Monounsaturated fatty acids } \\
\hline 14:1 & Myristoleic & 0.548 \\
\hline 15:1 & Pentadecenoic & 0.575 \\
\hline $16: 1$ & Palmitoleic & 0.871 \\
\hline $17: 1$ & Heptadecenoic & 0.449 \\
\hline 18:1 & Oleic & 0.588 \\
\hline $20: 1$ & Eicosenoic & 0.615 \\
\hline 22:1 & Erucic & -0.083 \\
\hline \multicolumn{3}{|c|}{ Polyunsaturated fatty acids } \\
\hline $16: 2$ & Hexadecadienoic & 0.577 \\
\hline $18: 2$ & Linoleic & 0.210 \\
\hline 18:3 & Octadecadienoic & 0.443 \\
\hline $20: 2$ & Eicosadicnoic & 0.695 \\
\hline
\end{tabular}




\begin{tabular}{|lll|}
\hline $20: 3$ & Eicosatrienoic & 0.326 \\
\hline $20: 4$ & Arachidonic & 0.769 \\
\hline $20: 5$ & Eicosapentaenoic & 0.387 \\
\hline $22: 3$ & Docosatrienoic & 0.319 \\
\hline $22: 4$ & Docosatetraenoic & 0.36 \\
\hline $22: 5$ & Docosapentaenoic & 0.365 \\
\hline $22: 6$ & Docosahexaenoic & 0.369 \\
\hline
\end{tabular}

* We analyzed only the principal component 1 with the greatest explanatory degree and listed its factor load. This principal component explained $33.2 \%$ of the variation in all 32 fatty acids.

Additionally, we evaluated the correlations between the "SFA" pattern with intakes of nutrients and food groups, results of which was shown in Supplement Table 2. The "SFA" pattern was positively associated with intake of protein, total fat, $(r=0.207,0.368$ respectively, all $p<0.001)$, but negatively related to fiber $(\mathrm{r}=-0.185, P<0.001)$. As for food groups, the "SFA" pattern was positively correlated to the intakes of fish, eggs, dairy and red meat $(r=0.372,0.320,0.283,0.282$, respectively, all $p<0.05)$, but negatively correlated with grain and vegetables $(r=-0.403,-0.100$, respectively, all $p<0.05)$.

\section{Association of the "SFA" pattern with risk of oral cancer}

Table 3 presents the $O R$ and $95 \% \mathrm{Cl}$ for oral cancer across the tertile categories for the "SFA" pattern. A positive association between the "SFA" pattern and risk of oral cancer was observed. In the crude model, those in the highest tertile of the "SFA" pattern had an increased risk of oral cancer compared with the lowest tertile, with a significant linear trend (OR=3.054; 95\% Cl. 2.184-4.265; $\left.P_{\text {trend }}<0.004\right)$. In model 1 , after adjusting for gender, age, marital status, residence, BMI, occupation and tumor history, the individuals in the highest tertile of the "SFA" pattern tended to have higher odds for oral cancer $\left(O R=2.874 ; 95 \% \mathrm{Cl}\right.$. 1.964-4.205; $\left.P_{\text {trend }}<0.001\right)$ compared with those in the lowest tertile. In model 2 , this result remained significant after further adjustment for lifestyle factors, including tobacco smoking, alcohol drinking and oral hygiene score $\left(O R=3.325 ; 95 \% \mathrm{Cl}\right.$. 2.222-4.975; $\left.P_{\text {trend }}<0.001\right)$. Moreover, this association was similarly observed when we treated the "SFA" pattern score as a continuous variable in the rude model, model 1 and model $2(O R=1.697,95 \% \mathrm{Cl}: 1.465-1.965 ; O R=1.652,95 \% \mathrm{Cl}$ : 1.402-1.947; $O R=$ 1.772, 95\% Cl: 1.490-2.106).

Table 3 The relation between the "SFA" pattern and oral cancer risk 


\begin{tabular}{|c|c|c|c|c|c|}
\hline \multirow[t]{2}{*}{ Model } & \multicolumn{3}{|c|}{ Tertiles of the "SFA" pattern score* } & \multirow[t]{2}{*}{$P_{\text {trend }}$} & \multirow{2}{*}{$\begin{array}{l}\text { "SFA" pattern } \\
\text { scoret }\end{array}$} \\
\hline & I & II & III & & \\
\hline case/control(n) & $138 / 196$ & $152 / 183$ & $144 / 190$ & & \\
\hline crude & 1.0(reference) & $\begin{array}{l}1.431(1.033- \\
1.984)\end{array}$ & $\begin{array}{l}3.054(2.187- \\
4.265)\end{array}$ & 0.001 & $\begin{array}{l}1.697(1.465- \\
1.965)\end{array}$ \\
\hline model1\# & 1.0(reference) & $\begin{array}{l}1.617(1.115- \\
2.344)\end{array}$ & $\begin{array}{l}2.874(1.964- \\
4.205)\end{array}$ & $\begin{array}{l}\square \\
0.001\end{array}$ & $\begin{array}{l}1.652(1.402- \\
1.947)\end{array}$ \\
\hline model2\# & 1.0(reference) & $\begin{array}{l}1.830(1.241- \\
2.698)\end{array}$ & $\begin{array}{l}3.325(2.222- \\
4.975)\end{array}$ & $\begin{array}{l}\square \\
0.001\end{array}$ & $\begin{array}{l}1.772(1.490- \\
2.106)\end{array}$ \\
\hline
\end{tabular}

*Three categories were obtained by tertiles of the fatty acid pattern score .Each participant was assigned a fatty acid pattern score.

\#Multivariable-adjusted Logistic regression models. Mode I adjusted for demographic characteristics: gender, age, marital status, residence, BMI, tumor, occupation, education.

Model 2 adjusted for demographic characteristics and tobacco smoking, drinking, oral hygiene score.

† Fatty acid pattern score treated as a continuous variable in the crude and two adjusted models

Furthermore, we visualized the association between the "SFA" pattern score and the risk of oral cancer using restricted cubic splines. The risk of oral cancer increased with the increase of "SFA" pattern score. However, the risk of oral cancer was relatively flat until around -0.68 of "SFA" pattern scores and then started to increase rapidly afterwards $\left(P_{\text {non-linearity }}=0.097\right)$ (Figure 2$)$.

\section{Stratification analysis between the "SFA" pattern and oral cancer risk}

Associations between the "SFA" pattern and oral cancer risk were stratified by demographic characteristics and life style exposure factors, results of which was shown in Figure 3. Positive association between oral cancer risk and the "SFA" pattern was observed in all subgroups except for the lower oral hygiene score group. There was no evidence of effect modification by sex, tobacco smoking, alcohol drinking, or oral hygiene score $\left(P_{\text {heterogeneity }}>0.1\right)$. The association varied across age (Figure 3; $\left.\mathrm{I}^{2}=87.8 \%, P_{\text {heterogeneity }}=0.004\right)$. The interaction was further tested by multiplying the "SFA" pattern score with age, and a multiplicative interaction was observed $\left(P_{\text {interaction }}<0.001\right)$.

\section{Discussion}

This hospital-based case-control study was conducted in Southeast China to illuminate the association between dietary fatty acids pattern and incidence of oral cancer. In the present study, we observed that the fatty acids intake varied widely between oral cancer patients and healthy controls. A "SFA" pattern 
was identified using the principal component analysis, and the "SFA" pattern was positively associated with risk of oral cancer.

Dietary fatty acids, especially for saturated fatty acid has long been reported to be associated with risk of cancers. Kim et al. performed a cross-sectional study, results of which showed that risk of colorectal cancer increased with higher SFA intake in Korean adults[29]. Several epidemiological studies have suggested increased consumption of SFA correlates with increased risk of prostate cancer and may be directly related to risk of biochemical recurrence and cancer progression[30-32]. However, there are also evidence supporting that dietary SFA are not associated with cancer risk or even negatively associated with cancer risk. Cao et al. performed a meta-analysis of prospective cohort studies, results of which showed that highest versus lowest levels of dietary SFA were not associated with the risk of breast cancer[33]. A meta-analysis of prospective cohort studies did not show an association between SFAs intake and colon cancer risk[34]. No associations were observed in the Nurses' Health Study cohort of dietary SFAs and epithelial ovarian cancer risk[35]. In the European Prospective Investigation into Cancer and Nutrition(EPIC), Aglago et al. found an inverse association between dietary total SFA and colorectal cancer[36]. To the best of our knowledge, reports of the association between dietary SFA and oral cancer are rare. A fatty acid pattern characterized by SFA was identified in our study and was found to be positively associated with oral cancer risk results of which was consist with some previous studies.

The major food sources of SFAs are of animal origin, including meat and dairy products. In fact, the association between dietary fatty acids and cancer risk may depend on types and food sources of fatty acids $[37,38]$. When we try to verify the identified "SFA" pattern using the correlation between the "SFA" pattern score with intakes of nutrients and food groups. We found that the intake of red meat and dairy was significantly higher in individuals with higher dietary "SFA" pattern scores. No studies have been conducted on the relationship between dietary saturated fatty acids driven from food sources and oral cancer. A study in Italy suggested that animal-derived foods such as dairy products and red meat could increase the risk of oral cancer[39]. Epidemiological evidence from Greece also indicates that meat products are positively associated with the risk of oral cancer[40]. In addition to the recognized etiologic factors in oral cancer, the relationship between food groups and "SFA" patterns (and the way they interact with "SFA" pattern) could affect the risk of oral cancer in our study. It is unclear whether the association between "SFA" pattern and oral cancer in our study are attributable to its food source or to unmeasured confounding remains unclear for now and warrants investigation.

In further analysis, by stratifying for demographic characteristics and life style exposure factors, we found that age may alter the relationship between the "SFA" pattern and oral cancer risk. Many previous studies have shown that age has an effect on cancer progression[41, 42]. In the present study, the "SFA" pattern were positively associated with oral cancer risk in both age subgroups, but the association was stronger in age groups younger than 49 years. Compared to MUFA and PUFA, SFA are more likely to come from red meat, processed meat and dairy products. Red meat is a primary source of the total amount of SFA, which has been identified as a dietary risk factor closely associated with various cancers [43, 44]. In addition to red meat, excessive intake of dairy products could also contribute to cancer risks [45]. So 
different food sources of SFAs may modulate the effect of SFAs on oral cancer risk. In our study, we found that compare with the older age group, the "SFA" pattern was more strongly associated with dairy products in the younger age group (Supplement Table 3). The results indicating that younger age groups may consume more saturated fatty acids from dairy products, such as cakes, cheese and ice cream bars, which may be positively associated with risk of oral cancer.

There are several limitations for this study. Firstly, this study was observational. Whereas we adjusted for a range of major oral cancer risk factors, residual confounding from imprecisely measured or unmeasured factors may be present. Secondly, this is a case-control study, since there is a long latency period which means that exposure data must be available for a period of several decades or more in order to identify true overall risks. The dietary fatty acids data was not collected prospectively, so dietary patterns may change over the long latency period, which may result potential bias. Lastly, the sample size of this study is limited and is collected from a single center, prospective study with large scale sample size is needed to verify the current findings.

\section{Conclusion}

In conclusion, the dietary fatty acid exposure based study provides support for a possible positive relationship between saturated fatty acids pattern and risk of oral cancer. In addition, potential interactions was found between saturated fatty acids pattern and age in oral cancer risk. Our findings support previous findings that there is suggestive evidence of a link between dietary pattern with head and neck cancer, but go beyond this by highlighting the role of specific fatty acid pattern in oral cancer susceptibility.

\section{Abbreviations}

OC: Oral cancer; SFA: saturated fatty acid; MUFA: monounsaturated fatty acid; PUFA: poly unsaturated fatty acid; FFA: trans fatty acid; FFQ: Food Frequency Questionnaire; PCA: principal component analysis; BMI: Body mass index; OR: Odds ratio; $\mathrm{Cl}$ : confidence interval

\section{Declarations}

\section{Ethics approval and consent to participate}

All the participants gave written informed consent to participate in the study. The present study was conducted in terms of the principles of the revised Declaration of Helsinki, which is a statement of ethical principles that directs physicians and other participants in medical research involving human subjects. The participants were assured about their anonymity and confidentiality of their information. Moreover, the study was approved by the Institutional Review Board of Fujian Medical University (Approval number: 2011053; Approval date: March 10, 2011).

\section{Consent for publication}


The Institutional Review Board of Fujian Medical University.

\section{Availability of data and materials}

Available from authors on reasonable request.

\section{Competing interests}

All authors report no conflict of interest in regard to this work.

\section{Funding}

The Provincial Colleges and Universities Program of Science and Technology Department of Fujian Province (No. JK2015017).

\section{Authors' contributions Conceptualization}

Yi Fan contributed to study design, compiled the data, analyzed the data, assisted in interpretation, and wrote the paper; Qing Chen contributed to study design, assisted in interpretation, and critically reviewed drafts of the paper; Sijie Wang contributed to study design, assisted in interpretation, and critically reviewed drafts of the paper; Yu Qiu conceived the study, contributed to study design, assisted in interpretation, and critically reviewed drafts of the paper. All authors have read and approved the manuscript.

\section{Acknowledgements}

The authors appreciate all people that patiently contributed to this study.

\section{References}

1. Chen W, Zheng R, Baade P, Zhang S, Zeng H, Bray F, et al. Cancer statistics in China,2015. CA Cancer J Clin. 2016;66(2).https://doi.org/10.3322/caac.21338.

2. Bray F, Ferlay J, Soerjomataram I, Siegel RL, Torre LA, Jemal A. Global cancer statistics 2018: GLOBOCAN estimates of incidence and mortality worldwide for 36 cancers in 185 countries. CA Cancer J Clin. 2018;68(6):394-424. http://dx.doi.org/10.3322/caac.21492.

3. Kadashetti V, Chaudhary M, Patil S, Gawande M, Shivakumar K, Patil S, et al. Analysis of various risk factors affecting potentially malignant disorders and oral cancer patients of Central India. J Cancer Res Ther. 2015;11(2):280-6. doi:10.4103/0973-1482.151417.

4. Hung L, Kung P, Lung C, Tsai M, Liu S, Chiu L, et al. Assessment of the Risk of Oral Cancer Incidence in A High-Risk Population and Establishment of A Predictive Model for Oral Cancer Incidence Using A Population-Based Cohort in Taiwan. Int J Environ Res Public Health. 2020;17(2):665. https://doi.org/10.3390/ijerph17020665. 
5. Radoi L, Paget-Bailly S, Cyr D, Papadopoulos A, Guida F, Schmaus A, et al. Tobacco smoking, alcohol drinking and risk of oral cavity cancer by subsite: results of a French population-based case-control study, the ICARE study. Eur J Cancer Prev. 2013;22(3):268-76. doi:10.1097/CEJ.0b013e3283592cce.

6. Gormley M, Dudding T, Sanderson E, Martin R, Thomas S, Tyrrell J, et al. A multivariable Mendelian randomization analysis investigating smoking and alcohol consumption in oral and oropharyngeal cancer. Nat Commun. 2020;11(1):6071. doi:10.1038/s41467-020-19822-6.

7. Chen F, He B, Yan L, Qiu Y, Lin L, Cai L. Influence of oral hygiene and its interaction with standard of education on the risk of oral cancer in women who neither smoked nor drank alcohol: a hospitalbased, case-control study. Br J Oral Maxillofac Surg;2017, 55(3):260-265. doi: 10.1016/j.bjoms.2016.11.316.

8. Rietbergen M, Leemans C, Bloemena E, Heideman D, Braakhuis B, Hesselink A, et al. Increasing prevalence rates of HPV attributable oropharyngeal squamous cell carcinomas in the Netherlands as assessed by a validated test algorithm. Int J Cancer. 2013;132(7):1565-71. https://doi.org/10.1002/ijc.27821.

9. Nair U, Bartsch H, Nair J. Alert for an epidemic of oral cancer due to use of the betel quid substitutes gutkha and pan masala: a review of agents and causative mechanisms. Mutagenesis. 2004;19(4):251-62. https://doi.org/10.1093/mutage/geh036.

10. Chen F, Yan L, Lin L, Liu F, Qiu Y, Wang J, et al. Dietary score and the risk of oral cancer: a casecontrol study in southeast China. Oncotarget. 2017;8(21):34610-6. doi:10.18632/oncotarget.16659.

11. Shivappa N, Hébert J, Rosato V, Garavello W, Serraino D, La Vecchia C. Inflammatory potential of diet and risk of oral and pharyngeal cancer in a large case-control study from Italy. Int $\mathrm{J}$ Cancer. 2017;141(3):471-9. doi:10.1002/ijc.30711.

12. Dalmartello M, Decarli A, Ferraroni M, Bravi F, Serraino D, Garavello W, et al. Dietary patterns and oral and pharyngeal cancer using latent class analysis. Int J Cancer. 2020;147(3):719-27. https://doi.org/10.1002/ijc.32769.

13. Perez-Cornago A, Huybrechts I, Appleby P, Schmidt J, Crowe F, Overvad K, et al. Intake of individual fatty acids and risk of prostate cancer in the European prospective investigation into cancer and nutrition. Int J Cancer. 2020;146(1):44-57. https://doi.org/10.1002/ijc.32233.

14. Dahm C, Gorst-Rasmussen A, Crowe F, Roswall N, Tjønneland A, Drogan D, et al. Fatty acid patterns and risk of prostate cancer in a case-control study nested within the European Prospective Investigation into Cancer and Nutrition. The American Journal of Clinical Nutrition. 2012;96(6):1354-61. https://doi.org/10.3945/ajcn.112.034157.

15. Matejcic M, Lesueur F, Biessy C, Renault A, Mebirouk N, Yammine S, et al. Circulating plasma phospholipid fatty acids and risk of pancreatic cancer in a large European cohort. Int $\mathrm{J}$ Cancer. 2018;143(10):2437-48. https://doi.org/10.1002/ijc.31797.

16. Ghamarzad Shishavan N, Mohamadkhani A, Ghajarieh Sepanlou S, Masoudi S, Sharafkhah M, Poustchi $\mathrm{H}$, et al. Circulating plasma fatty acids and risk of pancreatic cancer: Results from the Golestan Cohort Study. Clin Nutr. 2021;40(4):1897-904. doi:10.1016/j.clnu.2020.09.002. 
17. Aglago E, Murphy N, Huybrechts I, Nicolas G, Casagrande C, Fedirko V, et al. Dietary intake and plasma phospholipid concentrations of saturated, monounsaturated and trans fatty acids and colorectal cancer risk in the EPIC cohort. Int J Cancer. 2021;149(4):865-82. https://doi.org/10.1002/ijc.33615.

18. Nguyen S, Li H, Yu D, Cai H, Gao J, Gao Y, et al. Dietary fatty acids and colorectal cancer risk in men: A report from the Shanghai Men's Health Study and a meta-analysis. Int J Cancer. 2021;148(1):7789. doi:10.1002/ijc.33196.

19. Luu H, Cai H, Murff H, Xiang Y, Cai Q, Li H, et al. A prospective study of dietary polyunsaturated fatty acids intake and lung cancer risk. Int J Cancer. 2018;143(9):2225-37. https://doi.org/10.1002/ijc.31608.

20. Hu Frank B. Dietary pattern analysis: a new direction in nutritional epidemiology. Curr Opin Lipidol. 2002;13(1):3-9. doi:10.1097/00041433-200202000-00002.

21. Council NR. Diet and Health: Implications for Reducing Chronic Disease Risk. Washington(DC): The National Academies Press; 1989. https://doi.org/10.17226/1222.

22. Jacques $P$, Tucker KL. Are dietary patterns useful for understanding the role of diet in chronic disease? Am J Clin Nutr. 2001;73(1):1-2. doi:10.1093/ajcn/73.1.1.

23. Edefonti V, Bravi F, La Vecchia C, Randi G, Ferraroni M, Garavello W, et al. Nutrient-based dietary patterns and the risk of oral and pharyngeal cancer. Oral Oncol. 2010;46(5):343-8. https://doi.org/10.1016/j.oraloncology.2009.11.017.

24. Schulze M, Hoffmann K. Methodological approaches to study dietary patterns in relation to risk of coronary heart disease and stroke. Br J Nutr. 2006;95(5):860-9. doi:10.1079/BJN20061731.

25. Chen F, Wang J, Chen J, Yan L, Hu Z, Wu J, et al. Serum copper and zinc levels and the risk of oral cancer: A new insight based on large-scale case-control study. Oral Dis. 2019;25(1):80-6. doi:10.1111/odi.12957.

26. Chen F, He B, Yan L, Liu F, Huang J, Hu Z, et al. Tea consumption and its interactions with tobacco smoking and alcohol drinking on oral cancer in southeast China. Eur J Clin Nutr. 2017;71(4):481-5. doi:10.1038/ejcn.2016.208.

27. Willett W, Stampfer MJ. Total energy intake: implications for epidemiologic analyses. Am J Epidemiol. 1986;124(1):17-27. https://doi.org/10.1093/oxfordjournals.aje.a114366.

28. Sterne J, White I, Carlin J, Spratt M, Royston P, Kenward M, et al. Multiple imputation for missing data in epidemiological and clinical research: potential and pitfalls.BMJ. 2009; 338:b2393. https://doi.org/10.1136/bmj.b2393.

29. Kim J, Oh S, Kim Y, Kwon H, Joh H, Lee J,et al. Association between dietary fat intake and colorectal adenoma in korean adults: A cross-sectional study. Medicine. 2017;96:e5759. doi:10.1097/MD.0000000000005759.

30. Liss M, Al-Bayati O, Gelfond J, Goros M, Ullevig S, DiGiovanni J, et al. Higher baseline dietary fat and fatty acid intake is associated with increased risk of incident prostate cancer in the SABOR study. Prostate Cancer Prostatic Dis. 2019;22(2):244-51. doi:10.1038/s41391-018-0105-2. 
31. Bassett J, Severi G, Hodge A, MacInnis R, Gibson R, Hopper J, et al. Plasma phospholipid fatty acids, dietary fatty acids and prostate cancer risk. Int J Cancer. 2013;133(8):1882-91. https://doi.org/10.1002/ijc.28203.

32. Brasky T, Darke A, Song X, Tangen C, Goodman P, Thompson I, et al. Plasma phospholipid fatty acids and prostate cancer risk in the SELECT trial. J Natl Cancer Inst. 2013;105(15):1132-41. doi:10.1093/jnci/djt174.

33. Cao Y, Hou L, Wang W. Dietary total fat and fatty acids intake, serum fatty acids and risk of breast cancer: A meta-analysis of prospective cohort studies. Int J Cancer. 2016;138(8):1894-904. doi:10.1002/ijc.29938.

34. Kim M, Park K. Dietary Fat Intake and Risk of Colorectal Cancer: A Systematic Review and MetaAnalysis of Prospective Studies. Nutrients. 2018;10(12):1963. doi:10.3390/nu10121963.

35. Bertone ER, Rosner BA, Hunter DJ, Stampfer MJ, Speizer FE, Colditz GA, et al. Dietary fat intake and ovarian cancer in a cohort of US women. Am J Epidemiol. 2002;156(1):22-31. doi:10.1093/aje/kwf008.

36. Aglago EK, Murphy N, Huybrechts I, Nicolas G, Casagrande C, Fedirko V, et al. Dietary intake and plasma phospholipid concentrations of saturated, monounsaturated and trans fatty acids and colorectal cancer risk in the European Prospective Investigation into Cancer and Nutrition cohort. Int J Cancer. 2021; undefined(undefined), undefined. doi:10.1002/ijc.33615.

37. Gerber M. Background review paper on total fat, fatty acid intake and cancers. Ann Nutr Metab. 2009;55(1-3):140-61. doi:10.1159/000229000.

38. Thiebaut AC, Chajes V, Gerber M, Boutron-Ruault MC, Joulin V, Lenoir G, et al. Dietary intakes of omega- 6 and omega-3 polyunsaturated fatty acids and the risk of breast cancer. Int $\mathrm{J}$ Cancer. 2009;124(4):924-31. https://doi.org/10.1002/ijc.23980.

39. Barasch A, Litaker M. Nutrition and The Risk of Oral and Pharyngeal Cancer: The Evidence for Any Association Remains Weak and Clinical Significance Remains Limited. Journal of Evidence Based Dental Practice. 2012;12(null):263-4. doi:10.1016/S1532-3382(12)70050-7.

40. Petridou E, Zavras Al, Lefatzis D, Dessypris N, Laskaris G, Dokianakis G, et al. The role of diet and specific micronutrients in the etiology of oral carcinoma. Cancer. 2002;94(11):2981-8. https://doi.org/10.1002/cncr.10560.

41. DePinho RA. The age of cancer. Nature. 2000;408(6809):248-54. doi:10.1038/35041694.

42. Vincent-Chong VK, DeJong H, Rich LJ, Patti A, Merzianu M, Hershberger PA, et al. Impact of Age on Disease Progression and Microenvironment in Oral Cancer. J Dent Res. 2018;97(11):1268-76. https://doi.org/10.1177/0022034518775736.

43. Pascual JV, Rafecas M, Canela MA, Boatella J, Bou R, et al. Effect of increasing amounts of a linoleic-rich dietary fat on the fat composition of four pig breeds. Part l: Backfat fatty acid evolution. Food Chem. 2006;96(4):538-48. https://doi.org/10.1016/j.foodchem.2005.02.042.

44. de Lorgeril M, Salen P. New insights into the health effects of dietary saturated and omega- 6 and omega-3 polyunsaturated fatty acids. BMC Med. 2012;10:50. doi:10.1186/1741-7015-10-50. 
45. Parham GP. Dairy report one-sided: dairy products contribute to cancer risks. J Natl Med Assoc. 2005;97(4):582-3.

\section{Supplementary}

Supplementary Table 1 is not available with this version.

\section{Figures}




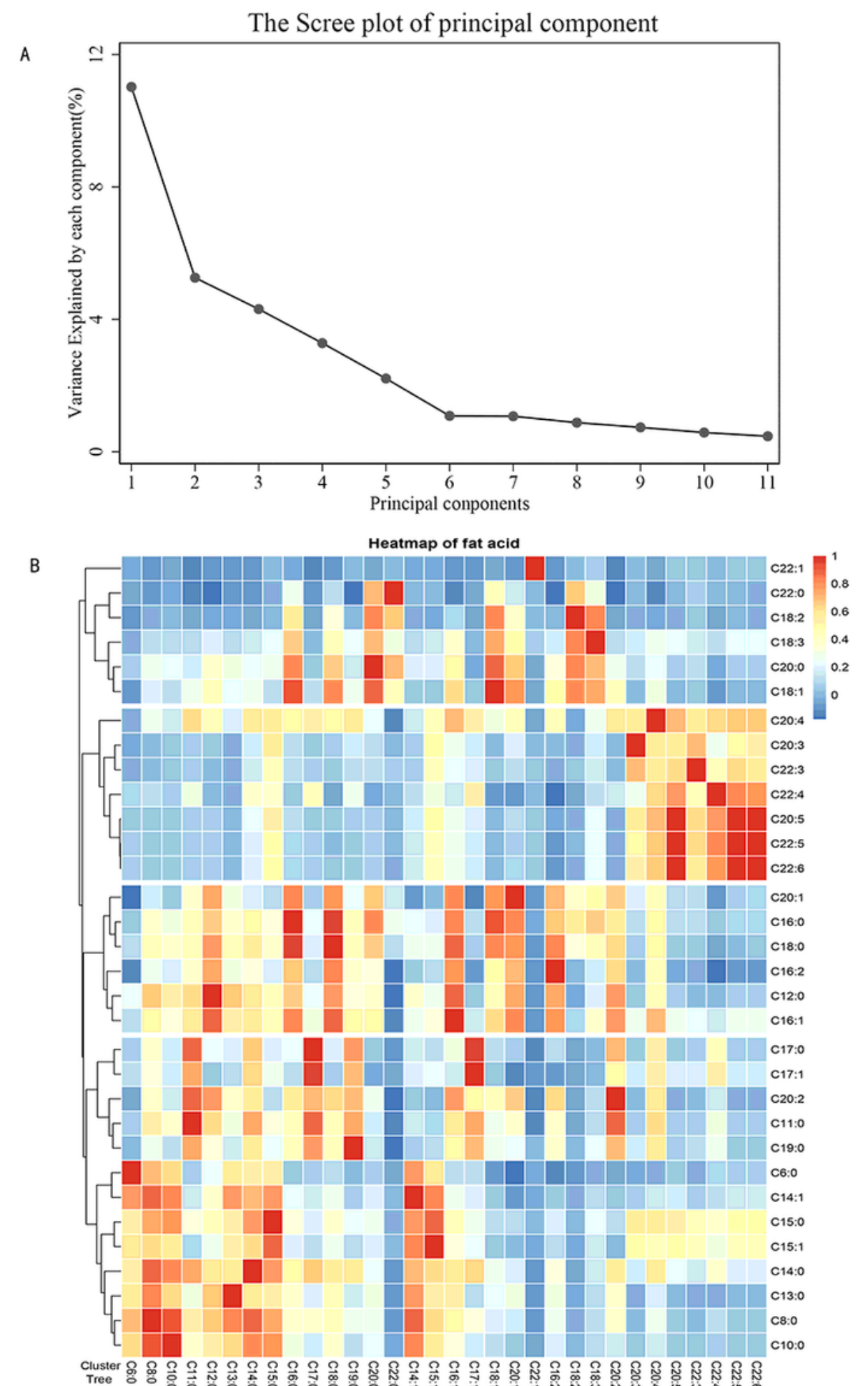

\section{Figure 1}

A figure caption was not provided with this version. 


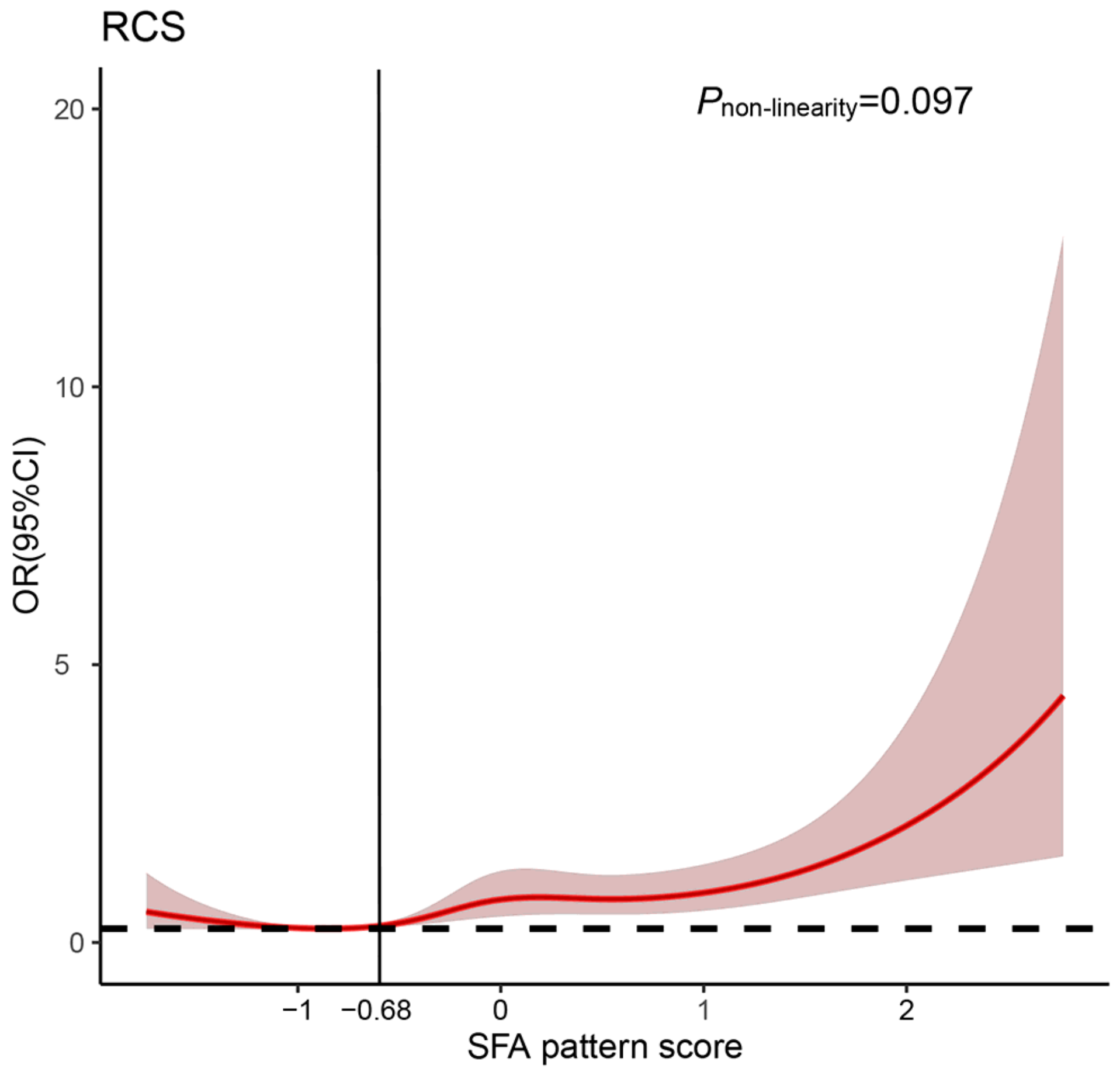

Figure 2

A figure caption was not provided with this version. 


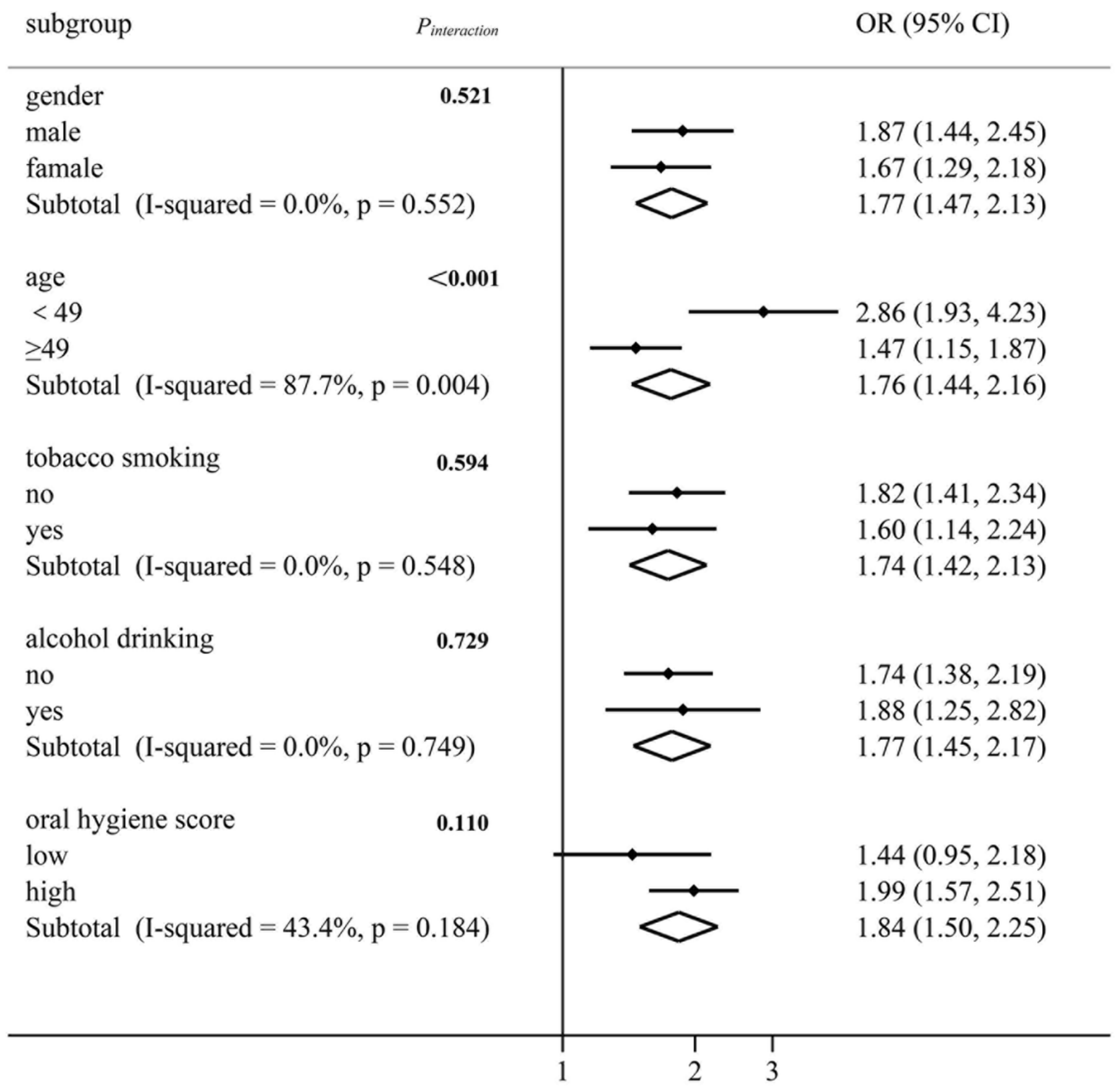

Figure 3

A figure caption was not provided with this version.

\section{Supplementary Files}

This is a list of supplementary files associated with this preprint. Click to download. 
- supplementfigure1.tif

- additionalfile2.docx 